Short communication

\title{
Development and experimental validation of a finite element model of total ankle replacement
}

\author{
Alexandre Terrier ${ }^{\mathrm{a}, *}$, Xabier Larrea ${ }^{\mathrm{a}}$, Jonas Guerdat ${ }^{\mathrm{a}}$, Xavier Crevoisier ${ }^{\mathrm{b}}$ \\ a Laboratory of Biomechanical Orthopedics, Ecole Polytechnique Fédérale de Lausanne, Station 19, 1015 Lausanne, Switzerland \\ ${ }^{\mathrm{b}}$ Service of Orthopaedics and Traumatology, University Hospital Center and University of Lausanne, Rue du Bugnon 46, 1011 Lausanne, Switzerland
}

\section{A R T I C L E I N F O}

\section{Article history:}

Accepted 16 December 2013

\section{Keywords:}

Ankle

Arthroplasty

Mobile-bearing

Finite element method

Stereo digital image correlation

\begin{abstract}
A B S T R A C T
Total ankle replacement remains a less satisfactory solution compared to other joint replacements. The goal of this study was to develop and validate a finite element model of total ankle replacement, for future testing of hypotheses related to clinical issues. To validate the finite element model, an experimental setup was specifically developed and applied on 8 cadaveric tibias. A non-cemented press fit tibial component of a mobile bearing prosthesis was inserted into the tibias. Two extreme anterior and posterior positions of the mobile bearing insert were considered, as well as a centered one. An axial force of $2 \mathrm{kN}$ was applied for each insert position. Strains were measured on the bone surface using digital image correlation. Tibias were CT scanned before implantation, after implantation, and after mechanical tests and removal of the prosthesis. The finite element model replicated the experimental setup. The first CT was used to build the geometry and evaluate the mechanical properties of the tibias. The second CT was used to set the implant position. The third CT was used to assess the bone-implant interface conditions. The coefficient of determination (R-squared) between the measured and predicted strains was 0.91 . Predicted bone strains were maximal around the implant keel, especially at the anterior and posterior ends. The finite element model presented here is validated for future tests using more physiological loading conditions.
\end{abstract}

(c) 2013 Elsevier Ltd. All rights reserved.

\section{Introduction}

The implant survival rate of total ankle replacement (TAR) is only $70-98 \%$ at $5-6$ years, $80-95 \%$ at $8-10$ years (Easley et al., 2011), and can drop to $45 \%$ at 15 years (Brunner et al., 2013). Failure causes include aseptic loosening, subsidence, cysts formation, peri-prosthetic and polyethylene fracture (Conti and Wong, 2001; Besse et al., 2009; Bonnin et al., 2011; Labek et al., 2011; Brunner et al., 2013).

Most current finite element models of TAR are limited to the intra-articular aspects or to the prosthetic components (Anderson et al., 2006, 2010; Reggiani et al., 2006; Espinosa et al., 2010; Barg et al., 2011). Therefore, the goal of this study was to develop and validate a finite element model of TAR, for future testing of hypotheses related to clinical issues.

\section{Methods}

\subsection{Experimental model}

Eight cadaveric tibias (77-90 years) perfused with a formaldehyde solution were used (Table 1). The tibias were CT scanned (GE Medical Systems) three times:

\footnotetext{
* Corresponding author. Tel.: +4121693 9498; fax: +41216938660.

E-mail address: alexandre.terrier@epfl.ch (A. Terrier).
}

before implantation, after implantation, and after the mechanical tests and removal of the prosthesis. Radio-opaque beads were fixed to the bone to register the 3 CTs. The CT resolution was $0.5 \times 0.5 \times 0.7 \mathrm{~mm}$. Bone quality was evaluated by the average Hounsfield number of the distal tibia, from the articular surface to $30 \mathrm{~mm}$ above it (Table 1 ).

An experienced surgeon inserted a non-cemented press fit tibial component (Salto, Tornier, Inc. Edina, MN, USA) according to manufacturer's recommendations. A distal tibial bone block was resected at a distance of $7 \mathrm{~mm}$ above the joint line, with a tibial posterior slope of 7 degrees. The tibia was cut at $150 \mathrm{~mm}$ from the implant plateau. The proximal part was fixed within an aluminium cylindrical support using 9 screws (Fig. 1). The tibia and screws were embedded in resin (MultiCast 20, Suter-Kunststoffe AG). The aluminium cylinder was fixed to the test plate of an Instron testing machine (ElectroPuls E3000). A block of polyethylene with same dimensions as the original mobile-bearing insert was placed on the plate of the tibial component. A universal joint was placed between the polyethylene and the load cell fixed to the Instron actuator. The proper alignment of the system was achieved with a mobile carriage supporting the aluminium cylinder. Three positions of the mobile-bearing insert were considered: centered, anterior and posterior. For the centered position, the mobile-bearing insert was aligned with the tibial plate and the loading axis. For the anterior and posterior positions, the mobile-bearing insert was displaced at the extreme end the tibial plate $(4.5 \mathrm{~mm})$. For the three positions, the axial loading force was $2 \mathrm{kN}$, applied at $1 \mathrm{~N} / \mathrm{s}$. A pre-conditioning of 50 cycles of $2 \mathrm{kN}$ at $0.1 \mathrm{~Hz}$ was performed before measurement.

The bone deformation was measured with stereo digital image correlation of 2 cameras (Limess Messtechnik \& Software $\mathrm{GmbH}$ ). The anterior side of the tibia, around the implant, was uniformly painted in white (spray) and then in black, to create the speckles required by the stereo-optical analysis. The measure was performed at maximum loading. The axial and transverse strains were derived from the displacement measurement (Vic-Snap Image Acquisition and Vic-3D Digital Image Correlation, Correlated Solutions, Inc., Columbia, USA). The systematic and 
random axial strain errors were evaluated using an extensometer (Instron 2620-603 Dynamic Extensometer) as a reference. The inclination of the implant during loading was assessed with the same stereo-optical technique.

The strains were evaluated in medial and lateral regions of interest (ROIs). The two ROIs were rectangles of $4 \mathrm{~mm}$ width and $12 \mathrm{~mm}$ high, centered at each sides of the implant fixation blade (Fig. 1). Within each ROI, the strains were estimated on a regular grid of 3 by 7 points, and then averaged, to avoid the lack of precision of a point-to point comparison.

\subsection{Numerical model}

The numerical model replicated the experimental setup. All tibias were segmented with the first CT, using imaging software Amira (http://www.vsg3d. com). Cortical and trabecular bone were segmented separately. Geometric models were built using Geomagic Studio (http://www.geomagic.com). The precise position of the implant position in the reconstructed tibia was obtained with the second $\mathrm{CT}$, which was registered to the first one using radio-opaque beads. The bone cuts and implant positioning was done with CAD software Solidworks (http:/ www.solidworks.com), under the supervision of the surgeon who performed the implantation on cadavers. The third CT was used to assess the bone cuts of the numerical model. The metallic tibial component was assumed rigid. Bone was nonhomogeneous and linear elastic. The elastic modulus was estimated from the first CT (Keller, 1994). The Poisson's ratio was 0.3.

The proximal part of the tibia embedded in resin was fully constrained. A point corresponding to the center of the universal joint in the experimental setup was rigidly linked to the implant (Fig. 2). An axial compressive force of $2 \mathrm{kN}$ was applied on this point. The rotation and axial displacement of this point were free, while its transverse translations were constrained. The centered, anterior, and posterior positions of the insert were replicated in the numerical model. The bone-implant interface was fully bonded. When the second CT presented a gap ( $>0.5 \mathrm{~mm}$ ) between the bone cut and the medial or lateral side of the plateau, no contact was considered at that side (Table 1 ).

Bone was meshed with quadratic 10-nodes tetrahedral elements. The average element size was $0.7 \mathrm{~mm}$ in the ROI, and higher elsewhere. The implant was meshed with rigid quadrilateral elements. A mesh sensitivity analysis was

Table 1

The 4 pairs of cadaveric tibia are reported with the sex and age of the donor. For the left (L) and right (R) side, we calculated the average Hounsfield (HU) number below the bone cut, and we evaluated if the contact between the bone ant the implant plate was either only lateral, only medial, of symmetric on both sides. The last column is the implant size.

\begin{tabular}{lllllll}
\hline Subject & Gender & Age & Side & HU & Contact & Size \\
\hline \multirow{2}{*}{1} & M & 90 & L & 259.9 & Symmetric & 1 \\
& \multirow{2}{*}{2} & & R & 240.6 & Symmetric & 1 \\
& F & 90 & L & 274.3 & Lateral & 0 \\
3 & \multirow{2}{*}{ M } & \multirow{2}{*}{82} & L & 259.0 & - & 0 \\
& & & R & 303.6 & Medial & 3 \\
4 & M & 77 & L & 409.4 & Symmetric & 3 \\
& & & R & 404.8 & Symmetric & 2 \\
\hline
\end{tabular}

performed with 5 different mesh refinements. The model was implemented in Abaqus v6.12 (http://www.simulia.com) and the analysis was performed with the implicit solver.

The longitudinal and transverse strains were evaluated at the same points as in the experiment. The comparison between the numerical predictions and the experimental measurements was performed for the longitudinal and transverse strains, the two ROIs, the three insert positions, and all tibias. The octahedral shear strain and the von Mises stress were calculated within the entire tibial bone. To evaluate the effect of the antero-posterior position of the polyethylene insert, the octahedral shear strain was evaluated along a line, within the symmetry plane of the implant, and at $1 \mathrm{~mm}$ from the implant distal end. We considered the maximal value, normalized with the value of the central position of the insert. Student's $t$-test with a significance level of $5 \%$ was used for all hypotheses testing.

\section{Results}

One tibia was excluded because of a bone fracture during implantation. The following results are based on the 7 remaining tibias. Systematic error of axial strain measurement was 140 microstrains. The measurement was adjusted to remove this bias. Random error was 230 microstrains. Implant inclination remained below 0.2 degree. Longitudinal and transverse strain ranged from

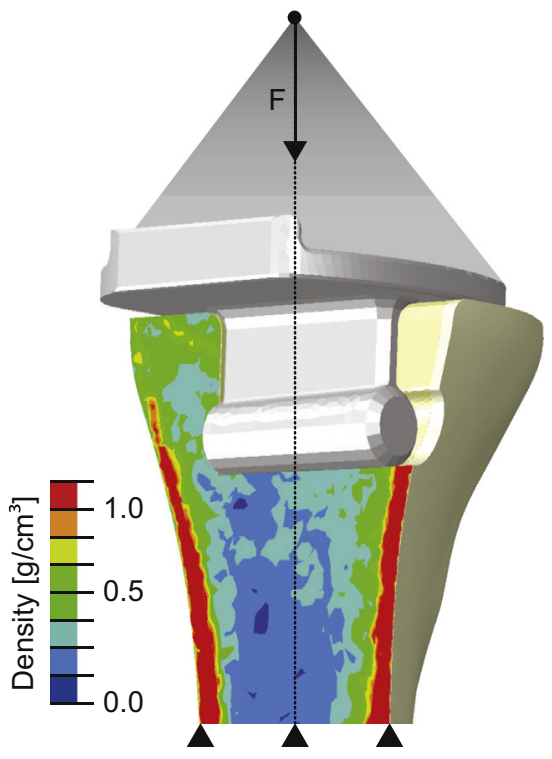

Fig. 2. Numerical model, showing the bone density distribution and the boundary conditions on a sagittal cut view of the tibia.
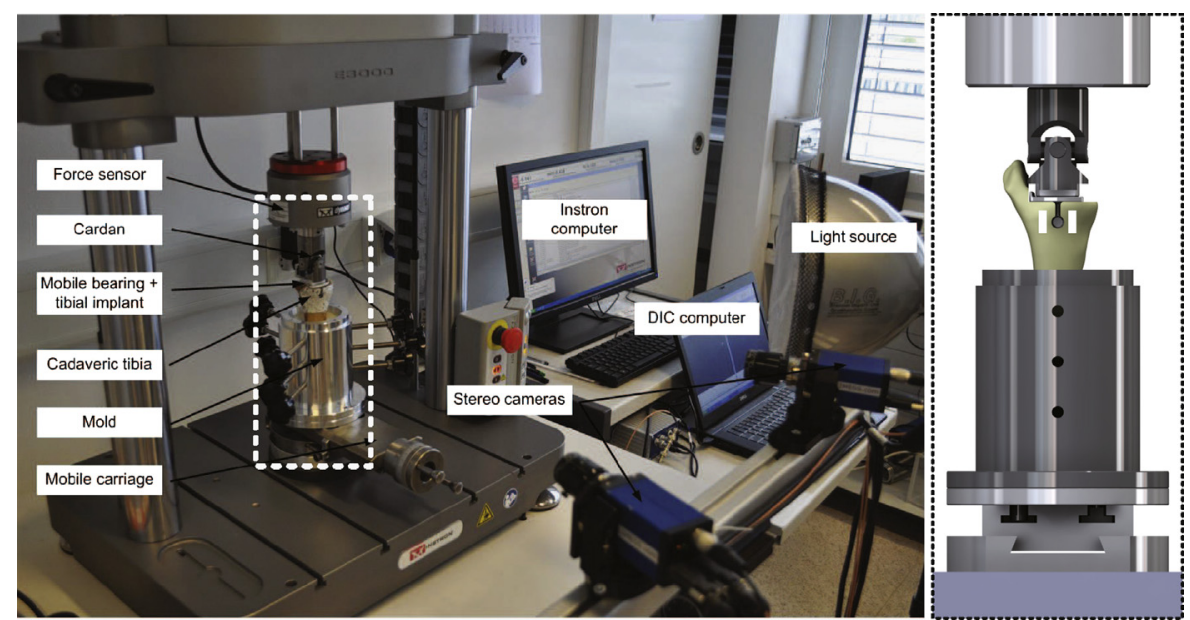

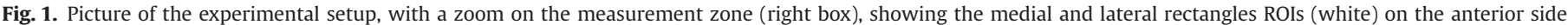
of the tibia. 
-2993 to 1277 microstrains. Medial and lateral ROIs were no statistically difference.

Predicted and measured strains were highly correlated (Fig. 3). Coefficient of determination was 0.91 , intercept was nearly zero $(-4.41 \mathrm{E}-5)$, slope was 0.80 , and root mean square error was 398 microstrains. The chosen FE mesh refinement altered the strain prediction by less than $2 \%$. For this FE mesh, the number of degrees of freedom was about 500,000.

Predicted octahedral shear strain within cortical bone was below 3000 microstrains (Fig. 4, left). Strain was maximal around the cylindrical part of the blade, at the anterior and posterior edges. The strain along a posterior-anterior line below the cylinder shape of the implant produced a similar pattern for all tibias, with two peaks and a plateau in-between (Fig. 4, right). For the centered position of the insert, the peak strain along this line ranged from 5200 to 21,500 microstrains among all tibias. The strain average along this line ranged from 2760 to 7150 microstrains. Compared to the centered position, the anterior and posterior position increased bone strain at the anterior, and respectively posterior side. Compared to the centered position, the eccentric positions increased the maximal strain under the implant keel, by less than $12 \%$. There was no statistical difference in strain between the two extreme positions and the centered one.

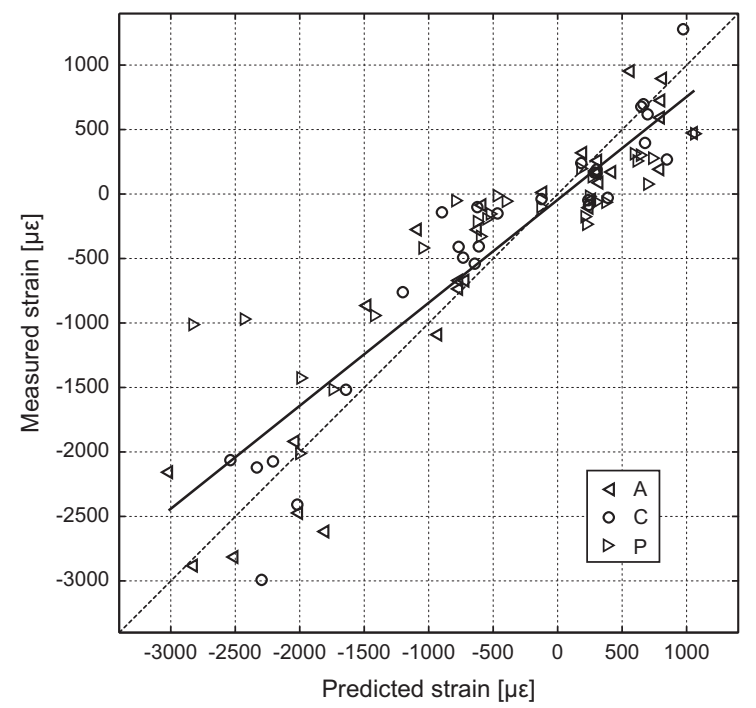

Fig. 3. Correlation between the experimental measurements and numerical predictions for all tibia and the anterior $(A)$, centered $(C)$ and posterior $(\mathrm{P})$ position of the mobile insert.
Among all tibias, von Mises stress peaks were mainly localized within cortical bone (Fig. 5). In trabecular bone, peak values (1 to $5 \mathrm{MPa}$ ) were localized between implant keel and cortical bone, and between implant plate and cortical bone.

\section{Discussion}

The relatively low survival rate of TAR, and its expected future revision occurrence are reported issues. There is thus an obvious need for improving midterm and long-term of TAR, and assessing revision prostheses. We proposed here a validated numerical model of TAR to further test hypotheses related to clinical problems, such as patient's selection, surgical technique, implant design and positioning, fixed bearing versus mobile-bearing prostheses.

The verification and validation of the numerical model was good. The measured strain on the tibia bone surface was consistent with the 3000 microstrains reported in the literature for typical activities (Yang et al., 2011; Al Nazeret al., 2012). The predicted strain within tibial bone exceeded this limit around the implant keel, for all tibias. The predicted strains suggest that bone microdamage might occur around the blade and at the border of the plate (Al Nazer et al., 2012). The strain values were higher when the average HU was lower, or when the bone support under the plate was not symmetrical. While the ratio between the highest average $\mathrm{HU}$ to the lowest was 1.7 , the corresponding average strain ratio under the keel was 2.3. The model predicted the expected stress concentration within cortical bone, and shielding between implant and cortical bone.

The eccentric cases simulated here covered reported extreme displacements of the mobile-bearing insert (Siegler et al., 2005; Reggiani et al., 2006; Leszko et al., 2008). The main limitation of the model was the use of non-fresh cadaveric bone, which were perfused and conserved 4 years in a formaldehyde solution. This may explain the correlation slope below 1 (Ohman et al., 2008). The validation process was performed only on the anterior side of the tibial surface. We have however observed a very limited bending of the system during the loading. Implant loading was simpler than in-vivo ankle forces. This was constrained by the experimental validation, but we assume that the loading was sufficiently close to the physiological conditions for future analyses with more physiological loads. Since the present model is based on linear elasticity, its validity is obviously limited to associated loading cases.

The present model offers great potential for future applications including better understanding of local biomechanics and problems
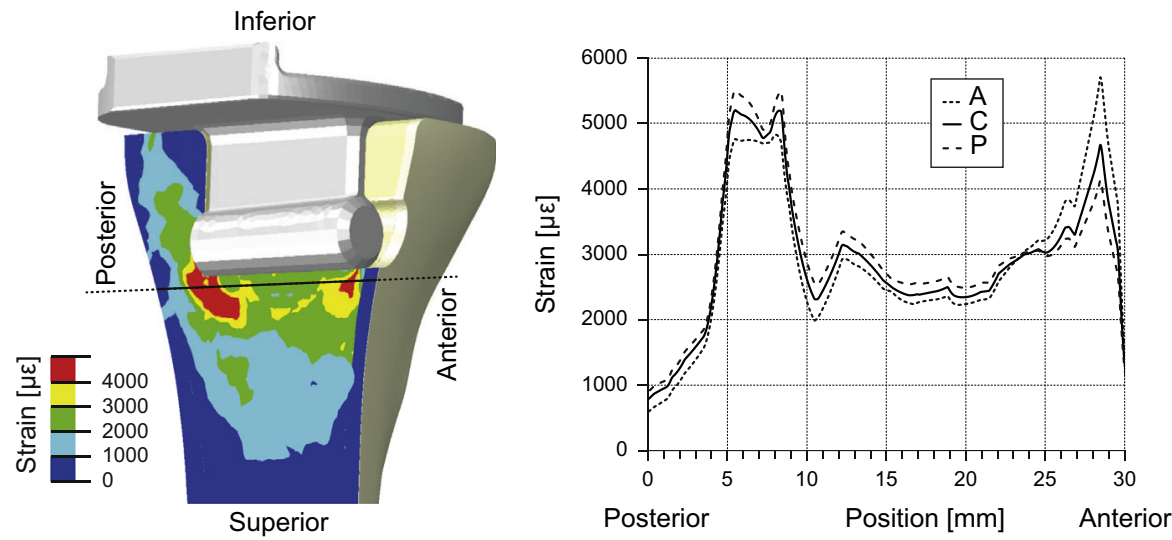

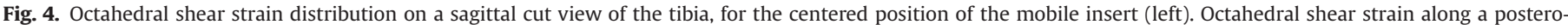
anterior line superior to the cylinder shape of the implant (right) for the anterior (A), centered (C) and posterior (P) position of the mobile insert. 


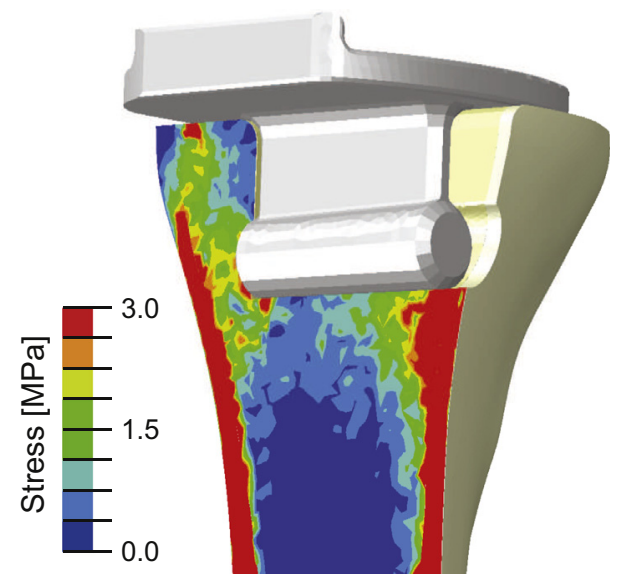

Fig. 5. Von Mises stress distribution on a sagittal cut view of the tibia.

associated with total ankle replacement, optimal implant design, fixation and positioning and development of revision implants.

\section{Conflict of interest statement}

None of the authors has any conflict of interest.

\section{Acknowledgements}

This study was financially supported by the Inter-institutional Center of Translational Biomechanics (EPFL-CBT). The cadaveric tibias were provided by the Department of Fundamental Neurosciences of the University of Lausanne. The CTs were performed at the "Centre Universitaire Romand de Médecine Légale" of the University of Lausanne. The implants and instrumentation were provided by Tornier (Tornier Inc., Edina, MN).

\section{References}

Al Nazer, R., Lanovaz, J., Kawalilak, C., Johnston, J.D., Kontulainen, S., 2012. Direct in vivo strain measurements in human bone-a systematic literature review. J. Biomech. 45 (1), 27-40.
Anderson, D.D., Goldsworthy, J.K., Shivanna, K., Grosland, N.M., Pedersen, D.R., Thomas, T.P., Tochigi, Y., Marsh, J.L., Brown, T.D., 2006. Intra-articular contact stress distributions at the ankle throughout stance phase-patient-specific finite element analysis as a metric of degeneration propensity. Biomech. Model. Mechanobiol. 5 (2-3), 82-89.

Anderson, D.D., Tochigi, Y., Rudert, M.J., Vaseenon, T., Brown, T.D., Amendola, A., 2010. Effect of implantation accuracy on ankle contact mechanics with a metallic focal resurfacing implant. J. Bone Joint Surg. Am. 92 (6), 1490-1500.

Barg, A., Elsner, A., Anderson, A.E., Hintermann, B., 2011. The effect of threecomponent total ankle replacement malalignment on clinical outcome: pain relief and functional outcome in 317 consecutive patients. J. Bone Joint Surg. Am. 93 (21), 1969-1978.

Besse, J.L., Brito, N., Lienhart, C., 2009. Clinical evaluation and radiographic assessment of bone lysis of the AES total ankle replacement. Foot Ankle Int. 30 (10), 964-975.

Bonnin, M., Gaudot, F., Laurent, J.R., Ellis, S., Colombier, J.A., Judet, T., 2011. The Salto total ankle arthroplasty: survivorship and analysis of failures at 7 to 11 years. Clin. Orthop. Relat. Res. 469 (1), 225-236.

Brunner, S., Barg, A., Knupp, M., Zwicky, L., Kapron, A.L., Valderrabano, V., Hintermann, B., 2013. The Scandinavian total ankle replacement: long-term, eleven to fifteen-year, survivorship analysis of the prosthesis in seventy-two consecutive patients. J. Bone Joint Surg. Am. 95 (8), 711-718.

Conti, S.F., Wong, Y.S., 2001. Complications of total ankle replacement. Clin. Orthop. Relat. Res. 391, 105-114.

Easley, M.E., Adams Jr., S.B., Hembree, W.C., DeOrio, J.K., 2011. Results of total ankle arthroplasty. J. Bone Joint Surg. Am. 93 (15), 1455-1468.

Espinosa, N., Walti, M., Favre, P., Snedeker, J.G., 2010. Misalignment of total ankle components can induce high joint contact pressures. J. Bone Joint Surg. Am. 92 (5), 1179-1187.

Keller, T.S., 1994. Predicting the compressive mechanical behavior of bone. J. Biomech. 27 (9), 1159-1168.

Labek, G., Klaus, H., Schlichtherle, R., Williams, A., Agreiter, M., 2011. Revision rates after total ankle arthroplasty in sample-based clinical studies and national registries. Foot Ankle Int. 32 (8), 740-745.

Leszko, F., Komistek, R.D., Mahfouz, M.R., Ratron, Y.A., Judet, T., Bonnin, M., Colombier, J.A., Lin, S.S., 2008. In vivo kinematics of the salto total ankle prosthesis. Foot Ankle Int. 29 (11), 1117-1125.

Ohman, C., Dall'Ara, E., Baleani, M., Jan, Van Sint, Viceconti, M., S., 2008. The effects of embalming using a $4 \%$ formalin solution on the compressive mechanical properties of human cortical bone. Clin. Biomech. (Bristol, Avon) 23 (10), 1294-1298.

Reggiani, B., Leardini, A., Corazza, F., Taylor, M., 2006. Finite element analysis of a total ankle replacement during the stance phase of gait. J. Biomech. 39 (8), 1435-1443.

Siegler, S., Udupa, J.K., Ringleb, S.I., Imhauser, C.W., Hirsch, B.E., Odhner, D., Saha, P. K., Okereke, E., Roach, N., 2005. Mechanics of the ankle and subtalar joints revealed through a 3D quasi-static stress MRI technique. J. Biomech. 38 (3), 567-578.

Yang, P.F., Brüggemann, G.P., Rittweger, J., 2011. What do we currently know from in vivo bone strain measurements in humans? J. Musculoskelet. Neuronal Interact. 11 (1), 8-20. 